\title{
Avoiding Bias of Focused Ion Beam Edge Resolution Measurements at High Doses
}

\author{
Zinovi Malamud, Yuval Greenzweig and Amir Raveh
}

Intel Israel (74), Ltd., Haifa, Israel

The Focused Ion Beam (FIB) has been an essential tool in the micro-electronics industry for many years, used for failure analysis, circuit modification, and analytical techniques [1,2]. The continual downscaling of minimum features in micro-electronics technology [3] has driven the need for finer machining, and thus FIB probe size $\left(d_{50}\right)$ has been steadily decreasing, recently reaching below $10 \mathrm{~nm}$ [4]. Several new "cold beam" ion sources are in research with some early implementations, which may eventually reduce FIB probe sizes significantly [5-9]. This development promises better imaging resolution and possibly better machining acuity, i.e., machining of finer features. To assess the goodness of such new ion sources, reliable measurement of beam probe size and beam profile are necessary. This paper addresses a challenge in probe size measurement which arises for small probe sizes.

FIB probe-size determination by resolution measurements presents the following challenge: The smallness of the features to be imaged, requires denser beam sampling, at a minimum dose which allows the signal to noise ratio (SNR) to enable resolution measurements [2,11]. Under such circumstances, samples erode quickly under the beam, introducing measurement artifacts, so that resolution samples and methods must be chosen with foresight. The prospect of yet smaller probes further heightens the importance of careful resolution analysis. We report herein a bias in resolution measurements, in which erroneously improved resolution is observed due to artifact depending on dose per scan. We present our recommendations on how to avoid this bias.

FIB probe size evaluation by edge resolution measurements is commonly performed on secondary electron (SE) images of samples with high contrast features. A typical sample consists of coarse gold particles on a carbon substrate, seen as bright inclusions on a dark background. Edge resolution is the corresponding distance perpendicular to the boundaries between the bright and the dark regions corresponding to brightness transitions, e.g. $20 \%$ - 80\%, or $35 \%$ - 65\%. Increasing imaging dose provides images with better SNR, and thus more reliable resolution measurements. However, excessive ion dose per image will erode and shrink the particles by sputtering. We use Tin-Ball on Carbon samples, for their better feature symmetry and uniformity. The vertical sidewalls of tin balls erode more slowly and evenly relative to gold particles, whose grain structure is less uniform. We noticed, however, that for tin balls, under high dose conditions, the edge contrast is not uniform over the ball perimeter. In Figure 1a a sharper edge is seen on the bottom of the ball, where the beam line scan exits the ball, and a more diffuse edge is seen on the top, where the beam enters the ball. The top image (a) was acquired with a higher dose of $0.028 \mathrm{nC} / \mu \mathrm{m} 2$, with a FIB having an established best $35 \%-65 \%$ edge resolution of $4.5 \mathrm{~nm}$ at $40 \mathrm{keV}$. In the polar graph (b) the best resolution on the bottom of the ball is surprisingly better than $3 \mathrm{~nm}$, and the resolution on the top is worse than $6 \mathrm{~nm}$. The lower image (c), with a lower dose of $0.007 \mathrm{nC} / \mu \mathrm{m}^{2}$, exhibits resolution isotropy, in the expected $4.5-5 \mathrm{~nm}$ range. The SE detector is annular and centered on the optical axis, and beam stigmation was unchanged between the images.

We measure resolution by a macro we wrote using ImageJ [12]. A short line segment drawn by the user defines the edge for the resolution measurement. An ImageJ function gives a linear array of pixel brightness values averaged over a user controlled line width typically set to 20 pixels ( $25 \mathrm{~nm}$ here). 
This array represents the brightness of the edge transition and is first fitted to a Fermi-Dirac function, after which a 35\%-65\% (or other) resolution is calculated. Due to the averaging, the program deals well with noisy images with local SNR as low as 2. This is useful since best FIB resolution is usually obtained for a smallest aperture, having low current and therefore poor SNR. Some automatic resolution programs erroneously mistake pixel to pixel brightness variation as representing very high resolution, which skews their statistical resolution result. Our program, albeit manual, does not have this limitation.
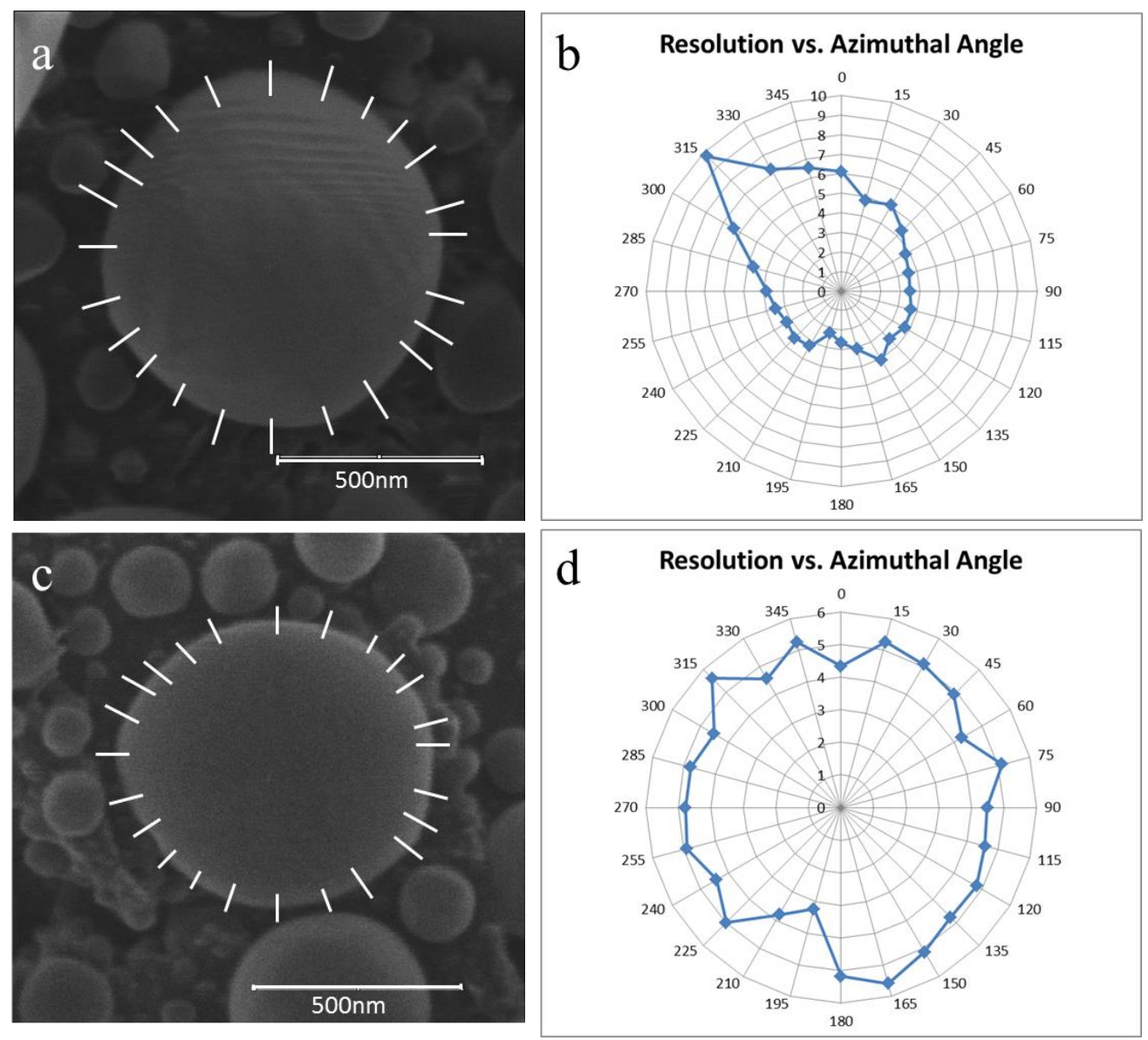

Figure 1. Tin ball SE images with a $4 \mathrm{X}$ larger dose on the top image $\left(0.028 \mathrm{nC} / \mu \mathrm{m}^{2}\right)$ (a), relative to the bottom image recommended dose $\left(0.007 \mathrm{nC} / \mu \mathrm{m}^{2}\right)(\mathrm{c})$. Line segments show the approximate locations of 24 edge resolution measurements on the balls' circumferences and corresponding edge resolution results $(35 \%-65 \%)$ are shown in the polar graphs to the right (b and d). For the high dose case (b), the resolution on the bottom of the sphere is more than $2 \times$ better (smaller) compared to the resolution on the top of the sphere, while for the lower dose case (d), the resolution is largely isotropic.

Our explanation for the anisotropy of the observed edge sharpness at high dose conditions (illustrated in Figure 2) is as follows: On the top edge of a ball, the scanning velocity (downwards) is aligned with the material edge recession velocity (also downwards), prolonging the edge transition time of the beam over the moving edge, and thereby erroneously worsening (enlarging) the image resolution. Conversely, these velocities are opposed where the beam exits the bottom of the tin ball, shortening the transition time, and making the resolution appear better-than-real. The above assumes that the beam scans horizontally across the sample and that line scans descend from the top to the bottom of the image. 


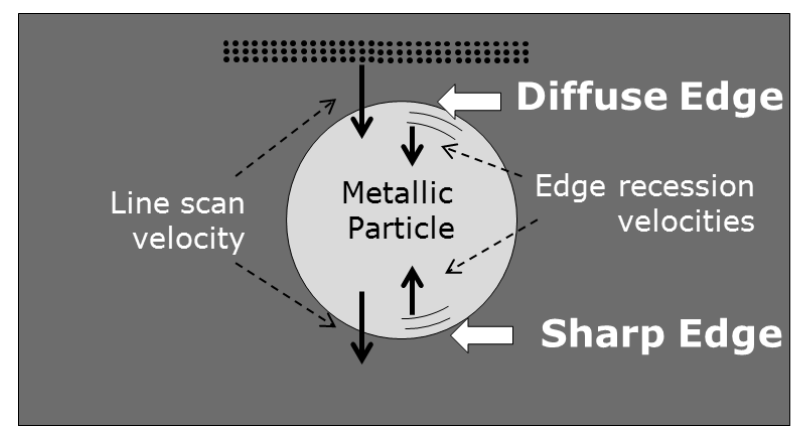

Figure 2. Explanation of the anisotropic resolution bias hinges on the scan velocity and edge recession velocity alignment vs. opposition. Resolution appears better where the two velocities are opposed (bottom, or trail edge), and appears worse where aligned (top, or lead edge).

We show resolution measurements from images of tin on carbon samples in Figure 3, at four dose values: $0.007,0.014,0.028$, and $0.056 \mathrm{nC} / \mu^{2}$. Balls of diameter between 700 and $800 \mathrm{~nm}$ were imaged. Measurements were performed on a Vectra-Visionary FEI system, ion energy $40 \mathrm{keV}$, current $2.6 \mathrm{pA}$, dwell time 4, 8, 16 and $32 \mu$ s respectively, and pixel size $1.22 \mathrm{~nm}$. The worse resolution on the highest dose image is not shown on the graph, being grossly off-scale. When limiting the dose per image to less than $0.007 \mathrm{nC} / \mu^{2}$, we observe little resolution anisotropy at different edges of the sample. However, for larger doses the resolution anisotropy increases roughly linearly with dose, and betterthan-real resolution is measured on the bottom of the spheres, as seen in the bottom data points.

To reinforce our understanding of this phenomenon of anisotropic resolution, we performed a simulation of material sputtering and SE emission by an ion beam passing once over a SE yielding round sample, to see if the phenomenon occurs for the modelled effects. We observe the simulated SE signal and calculate its resolution on the leading and trailing edges of the shape. The edge resolutions are plotted against the etch rate in Figure 3. The etch rate shows that top and bottom resolution are identical for low sputter rate, but resolution bifurcates for higher sputter rates.

The simulation is in 2-D, which is geometrically equivalent to a beam "line" approaching one side of top half a cylinder of radius 400nm (and parallel to its axis) moving over it and exiting the other side, which corresponds to our case where the ball diameter is much larger than the beam diameter. The beam was a double Gaussian: a narrow Gaussian with $\sigma=5.7 \mathrm{~nm}$ (corresponding to $4.4 \mathrm{~nm} 35 \%$ $65 \%$ resolution) representing the imaging probe-size, plus a $3 \times$ wider Gaussian representing beam tails, these Gaussians containing $80 \%$ and $20 \%$ of the current, respectively. Sputter rate dependence on angle of incidence was derived from TRIM. Ion incidence angle was calculated from the material surface slope at each time step, the normal direction sputter rate deduced from the above, and the vertical sputter rate then calculated. SE emission yield was also dependent on angle of ion incidence, modelling "edge brightening". No material re-deposition was accounted for, which is roughly justified for a convex sample. For clarification, the simulation was not "atomistic" but continuous, with spatial features on a grid of $0.01 \mathrm{~nm}$. The simulation ignores 3-D effects. Etch rate values were simulated, in the range $0.001-0.07 \mathrm{nC} / \mu \mathrm{m}^{2}$, and numbers were obtained for the top (leading) and bottom (trailing) edge resolutions vs. etch rate, and plotted as the dashed lines in Figure 3. The simulation reproduces 
the empirical resolution measurements results quite accurately for the bottom of the balls, while for the top of the balls, the actual measurement shows much worse values than that predicted by simulation.

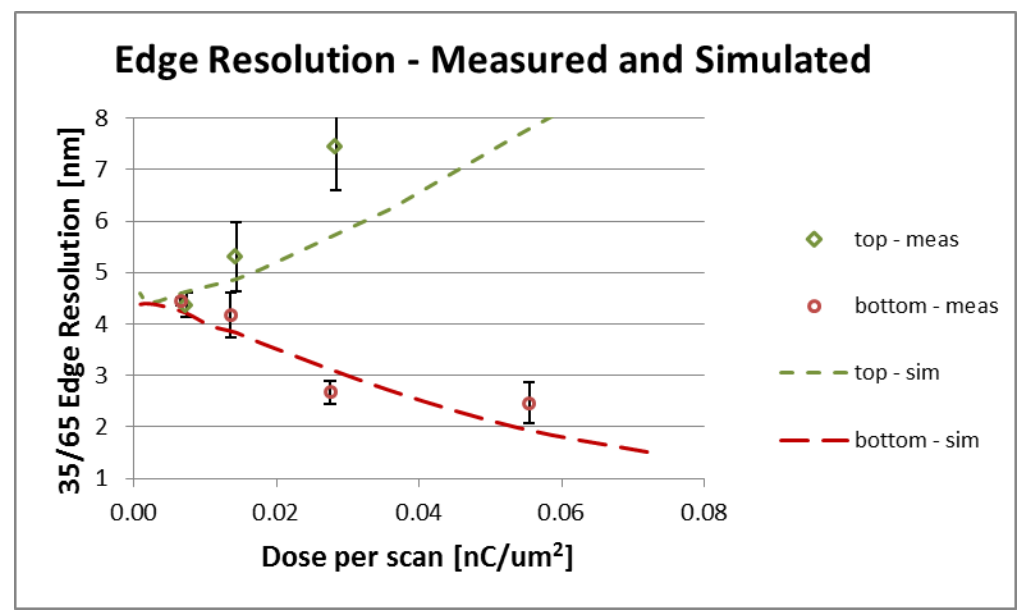

Figure 3. Results of a 2-D simulation of round shape sputtering and SE emission giving edge resolution for varying dose per scan. The bottom/top graph is for the "trailing"/"leading" edge. Erroneous resolution is demonstrated for higher dose per scan, as verified by the empirical data points.

We have demonstrated that FIB resolution measurements based on SE image edge resolution, give erroneously positive results beyond a critical dose per scan. In both empirical measurement and in simulation, we see that "bottom" edges of bright objects show better-than-real resolution, and "top" edges show worse resolution (assuming the beam scans across the FOV and then from top to bottom). For a pragmatic way to avoid this bias, we suggest measuring the resolution discrepancy (top vs. bottom), and if it is significant, reducing the dose to reach isotropy. For $30-40 \mathrm{keV} \mathrm{Ga}{ }^{+}$resolution measurements using tin on carbon samples, we recommend: 1) limiting dose per scan to $\sim 0.007 \mathrm{nC}_{\mu} \mathrm{m}^{2}$ 2) imaging balls of diameter greater than $\sim 700 \mathrm{~nm}$, whose edges are more sputter resistant. In the future, smaller beam probe sizes of heavier ions may necessitate new resolution samples, composed of lowsputter, high SE yield materials, with edge symmetries and consistency favourable for averaging.

\section{References:}

[1] I Utke, P Hoffmann and J Melngailis, J. Vac. Sci. Technol. B 26 (2008), p. 1197.

[2] M Utlaut in "Handbook of charged particle optics", ed. J Orloff, (CRC Press, New York) p. 523.

[3] G Moore, Electronics, 38 (1965), p. 114.

[4] J Gierak et al, J. Vac. Sci. Technol. B 15 (1997), p. 2373.

[5] DS Jun, VG Kutchoukov and P. Kruit, J. Vac. Sci. Technol. B 29 (2011), p. 06F603-1.

[6] B Knuffman, AV Steele and JJ McClelland, J. Appl. Phys. 114 (2013), 044303.

[7] L Kime et al, Phys. Rev. A 88 (2013), 033424.

[8] N Debernardi et al, J. Appl. Phys. 110, (2011) p. 024501.

[9] N Debernardi et al, New J. Phys. 14 (2012), 083011.

[10] S Tan et al, J. Vac. Sci. Technol. B 30 (2012), p. 06F606-1.

[11] V Castaldo, CW Hagen and P Kruit, Ultramicroscopy 111 (2011), p. 982.

[12] CA Schneider, WS Rasband and KW Eliceiri, Nat. Methods 9 (2012) p. 671. 